\title{
Coronal ejection and heating in variable X-ray sources (Research Note)
}

\begin{abstract}
W. Kluźniak
Nicolaus Copernicus Astronomical Center, ul. Bartycka 18, 00-716 Warszawa, Poland

e-mail:wlodek@camk.edu.pl

Received 1 August 2012 / Accepted 16 December 2012

ABSTRACT

A sudden increase in stellar luminosity may lead to the ejection of a large part of any optically thin gas orbiting the star. Test particles in circular orbits will become unbound and will escape to infinity (if radiation drag is neglected) when the luminosity changes from zero to at least one half the Eddington value, or more generally, from $L$ to $\left(L_{\mathrm{Edd}}+L\right) / 2$ or more. Conversely, a decrease in luminosity will lead to the tightening of orbits of optically thin fluid. Even a modest fluctuation of luminosity of accreting neutron stars or black holes is expected to lead to substantial coronal heating. Luminosity fluctuations may thus account for the high temperatures of the $\mathrm{X}$-ray corona in accreting black hole and neutron star systems.
\end{abstract}

Key words. accretion, accretion disks - scattering - X-rays: binaries - stars: winds, outflows - stars: neutron

\section{Introduction}

Low mass X-ray binaries (LMXBs) are bright X-ray sources whose luminosity is thought to be powered by accretion from a binary companion onto a neutron star or a black hole. Typically, each source can be found in one of two or more distinct spectral states, some of which include a hard X-ray power-law component interpeted as radiation from a hot $\left(\sim 10^{2} \mathrm{keV}\right)$ corona above the accretion disk (e.g., Done et al. 2007). Each state has an associated characteristic variability on many timescales. Nearly all sources exhibit quasi-random excursions of luminosity. In this paper, I examine some of the consequences of rapid variations of luminosity, particularly for optically thin Keplerian flow which I identify with the X-ray corona.

Since the paper of Walker \& Mészáros (1989) it is understood that high luminosity of an accreting neutron star will be associated with removal of angular momentum from the optically thin flow via the Poynting-Robertson drag. Many workers seem to assume that the primary effect of high luminosity is to bring matter down from its nearly-circular orbits towards the inner parts of the accretion disk and perhaps even onto the stellar surface. This is indeed true for optically thin flow in a steady radiation field. However, a rapid change in luminosity may have the opposite effect and, as I show below in a Newtonian calculation, under certain conditions may lead to ejection of the optically thin matter.

An even more important effect will occur when the luminosity undergoes a small but rapid change. The optically thin fluid will then continue its orbital motion in very nearly the same circular orbits, but will undergo strong heating caused by dissipation of excess kinetic energy. This may explain the presence of hot X-ray coronae in some spectral states of LMXBs.

The system under consideration is a standard thin accretion disk. The term "corona" is meant to signify the optically thin plasma "above the surface" of the disk. It is assumed that the dissipative processes responsible for angular momentum transport within the disk, and hence for accretion, occur in the optically thick regions "below the surface" of the disk. It is recognized that in some sources the optically thin plasma is much hotter than the body of the disk: spectral fits to the observed X-rays require the presence of a power-law component in addition to the multi-color thermal disk emission. The presence of such a hot corona is usually explained by magnetic heating, by analogy with the solar corona. However, I will not invoke the presence of magnetic fields to heat the corona. Instead, the heating will be related to the changes in luminosity that are assumed to occur, as observed in numerous X-ray binaries.

\section{Luminosity effects}

Consider orbits in the spherically symmetric Newtonian potential of a non-rotating star of mass $M$. If the star is radiating isotropically, optically thin hydrogen plasma suffers radiation pressure forces proportional to the Thomson cross-section and the radiative flux. At Eddington luminosity $L_{\text {Edd }}$, the radiative force balances gravity exactly. At lower luminosity, the presence of radiation pressure is equivalent to a proportional reduction of the gravitational mass by irradiation, so that the potential may be taken to be $V(r)=-G M(1-\lambda) / r$, with $\lambda \equiv L / L_{\text {Edd }}$. Hence, the eccentricity of a test-particle orbit is

$e=\sqrt{1+\frac{2 E l^{2}}{G^{2} M^{2}(1-\lambda)^{2}}}$,

where $E$ and $l$ are the specific (per unit mass) energy and angular momentum of the orbiting particle. The specific kinetic energy in circular orbit at $r_{0}$ for a source with luminosity $L$ and true gravitational mass $M$ is

$K_{\lambda}=\frac{G M(1-\lambda)}{2 r_{0}}$

Upon an impulsive change of stellar luminosity from zero to $L$, a particle that has been traveling in circular orbit at $r_{0}$ would 
conserve its kinetic energy and angular momentum so that its new orbit would be described by the following specific energy and angular momentum

$E=\frac{G M}{2 r_{0}}-\frac{G M(1-\lambda)}{r_{0}}, \quad l=l_{0}=\sqrt{G M r_{0}}$,

and because of the mismatch between energy and angular momentum values this orbit would no longer be circular. The eccentricity of the orbit would be, from Eq. (1),

$e=\frac{\lambda}{1-\lambda}$.

In particular, $\lambda \geq 1 / 2 \Rightarrow e \geq 1$, i.e., if the luminosity is now one half of the Eddington value, or greater, the particle is in an unbound orbit. If, on the other hand, the increase was from zero to less than one half of the Eddington luminosity, $\lambda<1 / 2 \Rightarrow$ $e<1$, the particle remains in a bound elliptic orbit with an increased semi-major axis $a=r_{0}(1-\lambda) /(1-2 \lambda)$.

\section{Coronal ejection}

In reality, the fluctuations of luminosity in LMXBs do not occur between $L=0$ and $L \neq 0$, but rather between some initial $L_{1} \neq 0$ and some final value $L \neq 0$. Generalizing the derivation from the previous section, we start with circular orbits at luminosity $L_{1}$, with $\lambda_{1} \equiv L_{1} / L_{\text {Edd }}$, the specific kinetic energy and angular momentum in circular orbits in the optically thin region being given by

$K_{1}=\frac{G M\left(1-\lambda_{1}\right)}{2 r}, \quad l_{1}=\sqrt{G M\left(1-\lambda_{1}\right) r}$.

Following an impulsive change of luminosity to $L$, the new orbits are defined by a new potential and the same values of kinetic energy and angular momentum

$V(r)=-\frac{G M(1-\lambda)}{r}, \quad K=K_{1}, \quad l=l_{1}$,

where, as before, $\lambda \equiv L / L_{\text {Edd }}$. The eccentricity of the new orbits is

$e=\frac{\left|\lambda-\lambda_{1}\right|}{1-\lambda}$

The condition for an unbound trajectory $(e \geq 1)$ now becomes

$1-\lambda \leq\left(1-\lambda_{1}\right) / 2$,

i.e.,

$L_{\mathrm{Edd}}-L \leq\left(L_{\mathrm{Edd}}-L_{1}\right) / 2$,

or

$\left(L_{\text {Edd }}+L_{1}\right) / 2 \leq L$.

So far, we have been considering test particles. However, the condition applies also to optically thin plasma. Since the wavefront of increased radiative flux travels at the speed of light, all elements of the plasma become unbound before any collisons on the outbound trajectories take place. The hot coronal plasma is assumed to be radiatively inefficient so any rarefaction or compression waves in an unbound medium cannot bind the bulk of it to the star (the total energy of the plasma is positive).

Identifying the corona with the optically thin region, the condition for its ejection would then be that the luminosity reduces its distance to the Eddington value by at least a factor of two. Clearly, the closer the initial value $L_{1}$ is to $L_{\text {Edd }}$, the lower the fractional value of luminosity increase needs to be for the corona to be ejected; e.g., an increase from $0.8 L_{\text {Edd }}$ to $0.9 L_{\text {Edd }}$ (corresponding to a fluctuation of less than $13 \%$ ) is sufficient to clear out the corona.

\section{Coronal heating}

Now we turn to changes of luminosity that do not lead to coronal ejection. This could be because the luminosity has increased from $L_{1}$ by less than $\left(L_{\mathrm{Edd}}-L_{1}\right) / 2$, thus violating Condition 10 , or because the luminosity has decreased. In any case, the new test particle orbit is an ellipse with semi-major axis $r_{0}(1-\lambda)$ / $\left|1+\lambda_{1}-2 \lambda\right|$ in the notation of the previous sections. The periastron is at radius $r_{0}$ for $\lambda>\lambda_{1}$ and at

$r_{-}=r_{0}\left(1-\lambda_{1}\right) /\left(1+\lambda_{1}-2 \lambda\right)$

for $\lambda<\lambda_{1}$. For a small decrease of luminosity, the latter value becomes $r_{-} \approx r_{0}\left[1-2\left(\lambda_{1}-\lambda\right) /\left(1-\lambda_{1}\right)\right]$.

Naturally, the state of the corona cannot be described by test particle orbits, as those of different particles would intersect. It is clear that some energy will be dissipated and the fluid orbits will circularize. The ultimate outcome is difficult to predict, because redistribution of angular momentum may occur. However, it seems clear that the dissipated energy will be on the order of $\left(\lambda_{1}-\lambda\right)^{2}$ times the virial energy of the corona.

Consider the evolution of a geometrically thin annulus initially orbiting at $r_{0}$, under a small impulsive change of luminosity of the central star $|\delta \lambda| \equiv\left|\lambda-\lambda_{1}\right| \ll 1$, and a subsequent dissipation of energy. If the annulus conserves its angular momentum, it will settle down in a circular orbit at $r_{1}=r_{0}\left(1-\lambda_{1}\right) /(1-\lambda)$ after dissipating an amount of specific energy equal to

$\delta E=\frac{(\delta \lambda)^{2}}{1-\lambda_{1}} \frac{G M}{2 r_{0}}$.

\section{Discussion}

The observed accreting neutron stars and black holes are usually quite luminous and are typically variable in time. It seems important to explore the consequences of rapid changes of luminosity whose magnitude may be a large fraction of the Eddington value.

The relevance of Poynting-Robertson drag to neutron stars was first pointed out by Walker \& Mészáros (1989). While there is no doubt that high luminosity is accompanied by radiation drag, which at least in the optically thin regime in a steady source will eventually remove kinetic energy and angular momentum of orbiting matter, this process takes time. If the luminosity undergoes a rapid change, the first and immediate response of orbiting matter is to change its trajectory.

I have assumed that the luminosity changes are either caused by changes in the mass-accretion flux that are unrelated to the state of the corona, or by a change of the state of the star, e.g., an X-ray burst. The opposite case, when the luminosity changes reflect accretion of the optically thin fluid, could correspond to oscillatory behavior and has been considered by Wielgus et al. (2012).

By considering Newtonian orbital mechanics, I have shown that an impulsive increase of central luminosity of sufficiently high magnitude $L-L_{1}>\left(L_{\mathrm{Edd}}-L_{1}\right) / 2$ may lead to an ejection of the optically thin corona on a dynamical timescale. This may have an application to X-ray spectral-state changes of black holes and neutron stars (e.g., Fender et al. 2005), and may be of some importance in X-ray bursts. Inclusion of radiation drag requires numerical computations, and will be postponed until another paper, where a fully general relativistic discussion will be presented (Stahl et al., in prep.).

The coronal response to small changes in luminosity was considered in Sect. 4. It seems inevitable that relatively minor changes in the luminosity of the central source lead to 
substantial energy dissipation. This could be the as yet unexplained mechanism of coronal heating. The estimated magnitude of the effect is surprisingly large. The dissipated energy at 50 Schwarzschild radii caused by a single excursion in luminosity of $10 \%$ Eddington $\left(0.1 L_{\text {Edd }}\right)$ is on the order of $10^{-4}$ of the coronal rest mass, i.e., it corresponds to a temperature of $\sim 100 \mathrm{keV}$. At $5 \mathrm{Schwarzschild} \mathrm{radii} \mathrm{the} \mathrm{same} \mathrm{result} \mathrm{will} \mathrm{be} \mathrm{ob-}$ tained by a fluctuation of only $3 \%$ Eddington luminosity.

In passing, we note that for a steady source of luminosity $\lambda$ in Eddington units, the orbital frequency in the optically thin regime is modified by a factor of $\sqrt{1-\lambda}$. Care must be taken to account for this effect when interpreting the redshift/blueshift of any spectral features in terms of orbital motion.

Acknowledgements. The anonymous referee is thanked for helpful comments that allowed a clarification of certain points.

\section{References}

Done, C., Gierliński, M., \& Kubota, A. 2007, A\&ARv, 15, 1

Fender, R., Belloni, T., \& Gallo, E. 2005, Ap\&SS, 300, 1

Walker, M. A., \& Mészáros, P. 1989, ApJ, 346, 844

Wielgus, M., Stahl, A., Abramowicz, M., \& Kluźniak, W. 2012, A\&A, 545, A123 